\title{
Effect of baicalin on hippocampal damage in kainic acid-induced epileptic mice
}

\author{
ZHENG-JIAN LIAO, RI-SHENG LIANG, SONG-SHENG SHI, CHUN-HUA WANG and WEI-ZHONG YANG \\ Department of Neurosurgery, Union Hospital, Fujian Medical University, Fuzhou, Fujian 350001, P.R. China
}

Received March 3, 2015; Accepted April 7, 2016

DOI: $10.3892 /$ etm.2016.3461

\begin{abstract}
The aim of the present study was to determine the effect of baicalin on the expression of miR-497 and its target B-cell lymphoma-2 (Bcl-2) in the hippocampus of kainic acid (KA)-induced epileptic mice. To establish status epilepticus (SE), $0.1 \mu \mathrm{g} / 5 \mu \mathrm{KA}$ was injected into the lateral cerebral ventricle in mice, which then received an intraperitoneal injection of baicalin $(100 \mathrm{mg} / \mathrm{kg})$ after 1 and $8 \mathrm{~h}$. Hematoxylin and eosin staining was used to observe the pathological changes in morphology and neuronal apoptosis was determined by terminal transferase-mediated dUTP nick end-labeling staining. Western blot analysis was used to detect the expression of Bcl-2 and cleaved caspase-3 proteins in the hippocampus, while reverse transcription-quantitative polymerase chain reaction was used to quantify hippocampal miR-497 expression. The results showed that baicalin significantly attenuated neuronal damage and apoptosis in the hippocampus $72 \mathrm{~h}$ after SE. In addition, baicalin decreased SE-induced expression of miR-497 and cleaved caspase-3 protein, while upregulating the expression of $\mathrm{Bcl}-2$ protein. In conclusion, the present results suggest that baicalin possesses potent antiapoptotic properties and attenuates hippocampal injury in mice after SE, which may be associated with the downregulation of miR-497 and cleaved caspase-3 and the upregulation of Bcl-2.
\end{abstract}

\section{Introduction}

Neuronal injury during status epilepticus (SE) is the result of increased excitotoxicity caused by calcium entrance into the cells leading to activation of various protease-mediated cascades and mitochondrial and nucleic injury, resulting in cell death (1).

Correspondence to: $\mathrm{Dr}$ Ri-Sheng Liang, Department of Neurosurgery, Union Hospital, Fujian Medical University, 29 Xinquan Road, Fuzhou, Fujian 350001, P.R. China

E-mail: rishengliang@yeah.net

Abbreviations: HE, hematoxylin and eosin; KA, kainic acid; SE, status epilepticus

Key words: baicalin, microRNA-497, B-cell lymphoma-2, caspase-3, apoptosis
Research have indicated that apoptosis plays a critical role in neuronal death following SE (2), in which caspase-3 is the final effector in the apoptotic signaling pathway $(3,4)$. In addition, other studies have demonstrated that the antiapoptotic protein B-cell lymphoma 2 (Bcl-2) protects against seizure-induced brain damage $(5,6)$. It is hypothesized that $\mathrm{Bcl}-2$ inhibits apoptosis by acting on the caspase- 3 dependent proteolytic cascade, and may attenuate SE-induced neuronal damage at least partially via the suppression of caspase-3 activity $(7,8)$.

MiRs are short non-coding RNAs that regulate gene expression at the post-transcription and/or translation levels $(9,10)$. MiR-497 is among the most prominently downregulated miRs in stroke-induced neuronal death and neurodegenerative disease $(11,12)$. Evidence suggests that miR-497 expression is inversely correlated with the regulation of apoptosis $(13,14)$, and has been shown to directly target Bcl-2 mRNA $(14,15)$. Additionally, a few groups have shown the involvement of miRs in the pathogenesis of epilepsy (16-18).

Baicalin is a flavonoid compound isolated from Scutellaria baicalensis Georgi, a traditional Chinese medicinal herb that is speculated to possesses anti-oxidative (19), anti-inflammatory (20), antiapoptotic (21) and anti-cancer properties (22). For example, baicalin has been shown to have a protective effect on permanent cerebral ischemia in rats through anti-inflammatory and antiapoptotic properties, possibly through the downregulation of nitric oxide synthase and cyclooxygenase- 2 mRNA and cleaved caspase- 3 protein expression (23). While a previous study showed that baicalin attenuates hippocampal injury after SE in mice (24), little is known about the mechanisms underlying this neuroprotective effect.

The present study investigated the role of cerebral miR-497 as a factor in the regulation of seizure-induced neuronal death after SE in mice. In addition, the study aimed to determine whether the neuroprotective effect of baicalin in this model may be associated with the downregulated expression of miR-497 and upregulation of the expression of a known, antiapoptotic target gene, $\mathrm{Bcl}-2$, which results in reduced caspase-3 cleavage.

\section{Materials and methods}

Experimental animals. A total of 99 adult ICR male mice weighing 22-28 g (Shanghai Laboratory Animal Center, Chinese Academy of Sciences, Shanghai, China) were used 
in this study. All animals were housed using an alternating 12 -h light/dark cycle in a temperature $\left(18-25^{\circ} \mathrm{C}\right)$ and humidity (50-60\%) controlled environment. Food and water were available ad libitum. The mice were allowed to adapt to laboratory conditions for at least 1 week before starting the experiment. Mice were divided into three groups as follows: i) Sham control group ( $\mathrm{n}=33$ ), which underwent sham operation and received vehicle (Beijing Zhongshan Golden Bridge Biotechnology Co., Ltd.); ii) SE group (n=33), which was subjected to kainic acid (KA) injection and received vehicle; and iii) baicalin group $(n=33)$, which was subjected to KA injection and treated with baicalin $100 \mathrm{mg} / \mathrm{kg}$. This study was approved by the Institutional Animal Care, Ethics and Use Committees of Fujian Medical University (Fuzhou, China).

Seizure induction. All surgical procedures were carried out in accordance with the National Institute of Health Guide for the Care and Use of Laboratory Animals and performed using sterile/aseptic techniques in accordance with institutional guidelines. Focal-onset SE was induced by intracerebral ventricle stereotaxic microinjection of KA (Sigma-Aldrich, St. Louis, MO, USA), and was performed as previously described (25). Briefly, mice were anesthetized with an intraperitoneal injection of chloral hydrate ( $300 \mathrm{mg} / \mathrm{kg}$; Sigma-Aldrich) and placed in a stereotaxic frame (Gilson, Inc., Villiers le Bel, France). Following a midline scalp incision, the bregma was located. The skull was then drilled for placement of a guide cannula (bregma coordinates; $\mathrm{AP}=1.9 \mathrm{~mm}, \mathrm{~L}=2.1 \mathrm{~mm}$ ) based on a mouse brain stereotaxic atlas (26). Then, the animal was restrained while an injection cannula was lowered $2.4 \mathrm{~mm}$ below the brain surface into the lateral ventricle for injection of KA. The injected dose was $0.8 \mu \mathrm{g} \mathrm{KA}$ in $5 \mu \mathrm{l}$ normal saline at $1 \mu \mathrm{l} / \mathrm{min}$, to elicit a prolonged SE. Non-seizure control mice underwent the same surgical procedures but received intracerebral ventricle vehicle (normal saline, $5 \mu 1$ ). During the surgery, rectal temperature was controlled at $37^{\circ} \mathrm{C}$ using a heating pad and heat lamp (Beijing Cinontech Co., Ltd., Beijing, China; http://www.ysf2005.bioon.com.cn/product_606.html). All efforts were made to minimize the number of animals used and to avoid pain and suffering. In all cases, Racine stage 5 (rearing and falling) seizures were observed within $1 \mathrm{~h}$ following KA administration (27).

Drug administration. Baicalin (purity, >95\%; CAS Number: 21967-41-9; Sigma-Aldrich) was dissolved in normal saline and injected intraperitoneally into mice assigned to the baicalin group at 1 and $8 \mathrm{~h}$ after the onset of SE. Mice in the SE group were injected with normal saline at the same volume and time points as the baicalin group.

Preparation of brain tissue slices for hematoxylin and eosin (HE) staining. A total of 18 mice were selected for HE staining (Beyotime Institute of Biotechnology, Shanghai, China) (6 per group), and were anesthetized using chloral hydrate $(10 \%, 3.5 \mathrm{ml} / \mathrm{kg}$, i.p.), then sacrificed $72 \mathrm{~h}$ after the onset of SE using a transcardiac infusion of normal saline followed by $4 \%$ paraformaldehyde to fix the brain. The mice were then decapitated and the fixed brains were removed and embedded in paraffin. The brain was then cut into 5-6 $\mathrm{mm}$ thick sections containing the hippocampus, which were further cut with a HM340E microtome (Microm International $\mathrm{GmbH}$, Walldorf, Germany) into $30 \mu \mathrm{m}$ thick coronal sections. Then, the sections were deparaffinized with xylene (Guangzhou Chemical Reagent Factory, Guangzhou, China) and rehydrated with graded alcohol. Subsequently, the slices were stained using HE, mounted to glass slides and coverslipped using neutral gum (ZLI-9516; Zhongshan Golden Bridge Biotechnology Co., Ltd.).

Terminal transferase-mediated dUTP nick end-labeling (TUNEL) analysis. TUNEL staining was carried out using the in situ cell death detection kit (Promega Corporation, Fitchburg, WI, USA) according to the manufacturer's instructions. Briefly, 18 mice (6 per group) were anesthetized, then sacrificed at $72 \mathrm{~h}$ following onset of SE by transcardiac perfusion of normal saline followed by $4 \%$ paraformaldehyde. The animals were then decapitated with brain tissue removed and processed as shown above. However, in addition to the aforementioned procedure, after deparaffinizing the sections were digested using proteinase $\mathrm{K}$ (Zhongshan Golden Bridge Biotechnology Co., Ltd.). The slides were placed in the equilibration buffer and then incubated in TdT enzyme (Promega Corporation) at $37^{\circ} \mathrm{C}$ for $60 \mathrm{~min}$, followed by 2xSSC (Zhongshan Golden Bridge Biotechnology Co., Ltd.). to stop the reaction. Endogenous peroxidase activity was then blocked with $0.3 \% \mathrm{H}_{2} \mathrm{O}_{2}$ and the sections were incubated in a streptavidin horseradish-peroxidase solution (Zhongshan Golden Bridge Biotechnology Co., Ltd.) for $30 \mathrm{~min}$ at room temperature. 3,3'-Diaminobenzidine (Zhongshan Golden Bridge Biotechnology Co., Ltd.) was used as chromogen and the sections were counterstained with hematoxylin (Zhongshan Golden Bridge Biotechnology Co., Ltd.). The sections were then mounted on glass slides and coverslipped using neutral gum (ZLI-9516; Zhongshan Golden Bridge Biotechnology Co., Ltd.). The TUNEL-positive cells and total cells in the hippocampal CA3 subfields were manually counted while viewed at $\mathrm{x} 400$ magnification using a microscope (Olympus Corporation, Tokyo, Japan). The number of TUNEL-positive cells was expressed as the percentage of total counted cells.

Immunohistochemistry. A total of 18 mice (6 per group) were anesthetized $72 \mathrm{~h}$ following SE induction then sacrificed by transcardiac perfusion of normal saline followed by $4 \%$ paraformaldehyde. Decapitation was performed, with the brains removed and processed as shown above. However, the paraffin-embedded sections were deparaffinized with xylene and rehydrated graded alcohol, followed by treatment with $1 \%$ hydrogen peroxide to eliminate endogenous peroxidase activity. After treatment with 5\% goat serum reagent (Zhongshan Golden Bridge Biotechnology Co.,Ltd.) at room temperature for $30 \mathrm{~min}$, the sections were then incubated overnight at $4^{\circ} \mathrm{C}$ with a rabbit polyclonal anti-Bcl-2 antibody (1:50; ZA-0536; Zhongshan Golden Bridge Biotechnology Co., Ltd.). The sections were incubated with biotinylated goat anti-rabbit secondary antibody (1:200; ZDR-5403; KPL, Inc., Zhongshan Golden Bridge Biotechnology Co., Ltd.) at room temperature for $60 \mathrm{~min}$, followed by incubation with a streptavidin-biotin peroxidase complex solution (Wuhan Boster Biological Technology, Ltd., Wuhan, China) at room temperature for $120 \mathrm{~min}$. Subsequently, the sections were stained using 3,3'-diaminobenzidine, 

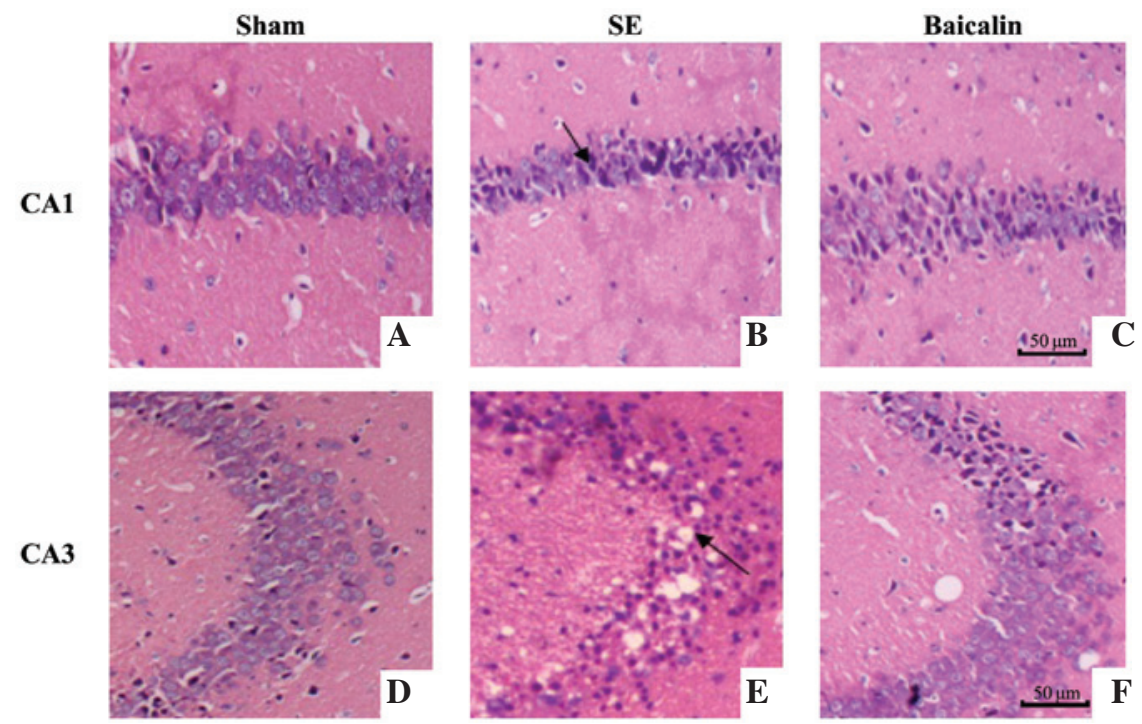

Figure 1. Photomicrographs showing cell morphology $72 \mathrm{~h}$ following status epilepticus (SE) induction (magnification, x200; stain, hematoxylin and eosin). Regions within the hippocampal (A-C) CA1 and (D-F) CA3 subfields shown at magnification, x200. (A and D) Neurons from sham animals and (B and E) neurons from seizure-induced injury. (A) Arrows in 1a indicates shrunken neurons. (E) Arrow indicates an empty space known as cavitation, implying the formation of vapor cavities. (C and F) HE staining indicated that SE-induced neuronal injury in the hippocampal CA1 and CA3 subfields were markedly reduced by $100 \mathrm{mg} / \mathrm{kg}$ baicalin. The arrow in $1 \mathrm{~B}$ indicates shrunken neurons.
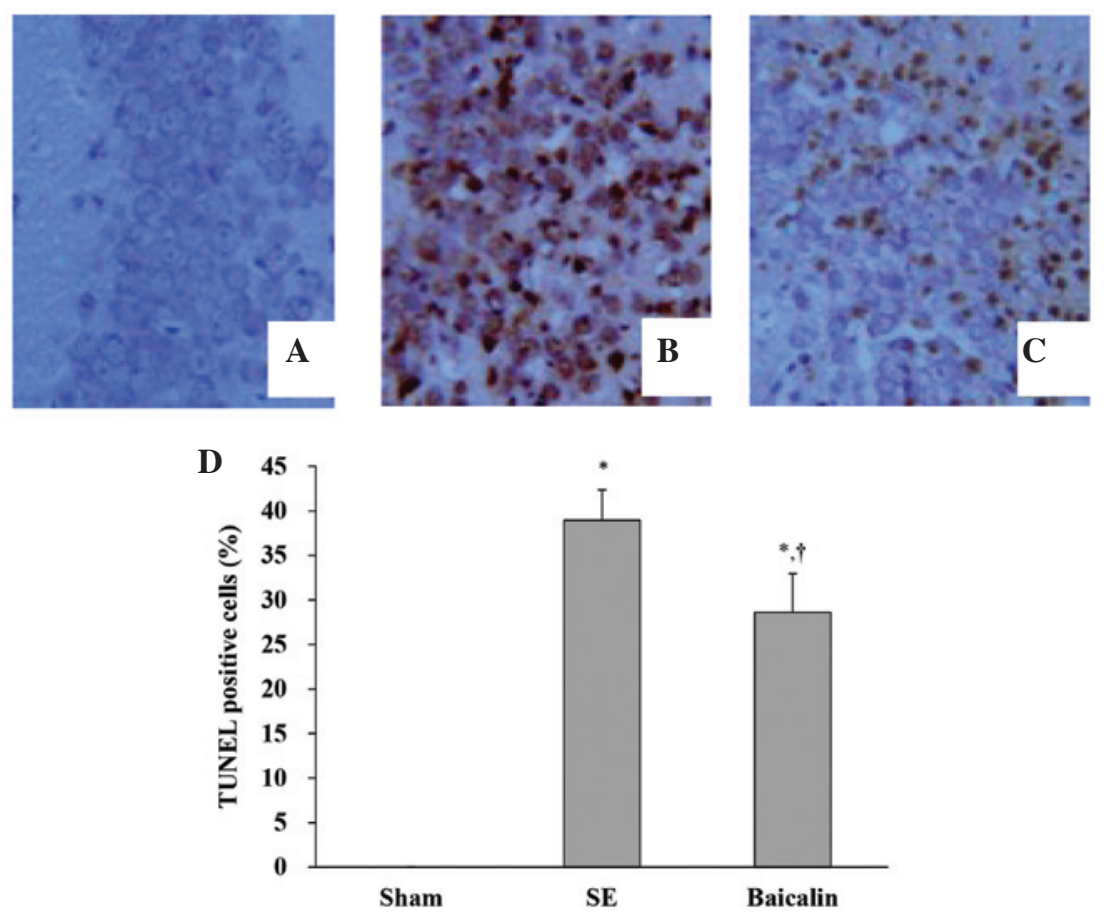

Figure 2. Neuronal apoptosis in hippocampal CA3 subfields showing $72 \mathrm{~h}$ following induction of SE. Representative images of (A) sham, (B) SE and (C) baicalin groups (magnification, $\mathrm{x} 4,000$ ). (D) Graph showing the percentage of TUNEL-positive cells for each treatment group expressed as mean \pm standard deviation (n=6 per group). ${ }^{*} \mathrm{P}<0.05$ vs. sham group. ${ }^{\dagger} \mathrm{P}<0.05$ vs. SE group. SE, status epilepticus.

counterstained with hematoxylin, dehydrated, then mounted and coverslipped using neutral gum (ZLI-9516; Zhongshan Golden Bridge Biotechnology Co., Ltd.). All sections were processed with Image-Pro Plus (Media Cybernetics, Inc., Rockville, MD, USA) for imaging and analysis.

Western blot analysis. For western blot analysis, 18 mice (6 per group) were anesthetized at $72 \mathrm{~h}$ following the induction of SE, sacrificed via transcardiac perfusion of normal saline followed by $4 \%$ paraformaldehyde, then decapitated for brain removal. The hippocampus was dissected, homogenized and purified using protein extraction reagents according to the manufacturer's instructions (KeyGen Biotech, Co., Ltd., Nanjing, China). Protein concentration was determined by the Bradford method using bovine serum albumin (Beyotime Institute of Biotechnology) as the standard. Different samples with an equal quantity of protein $(20 \mathrm{mg})$ were separated on $10 \%$ SDS-polyacrylamide gels (Wuhan 

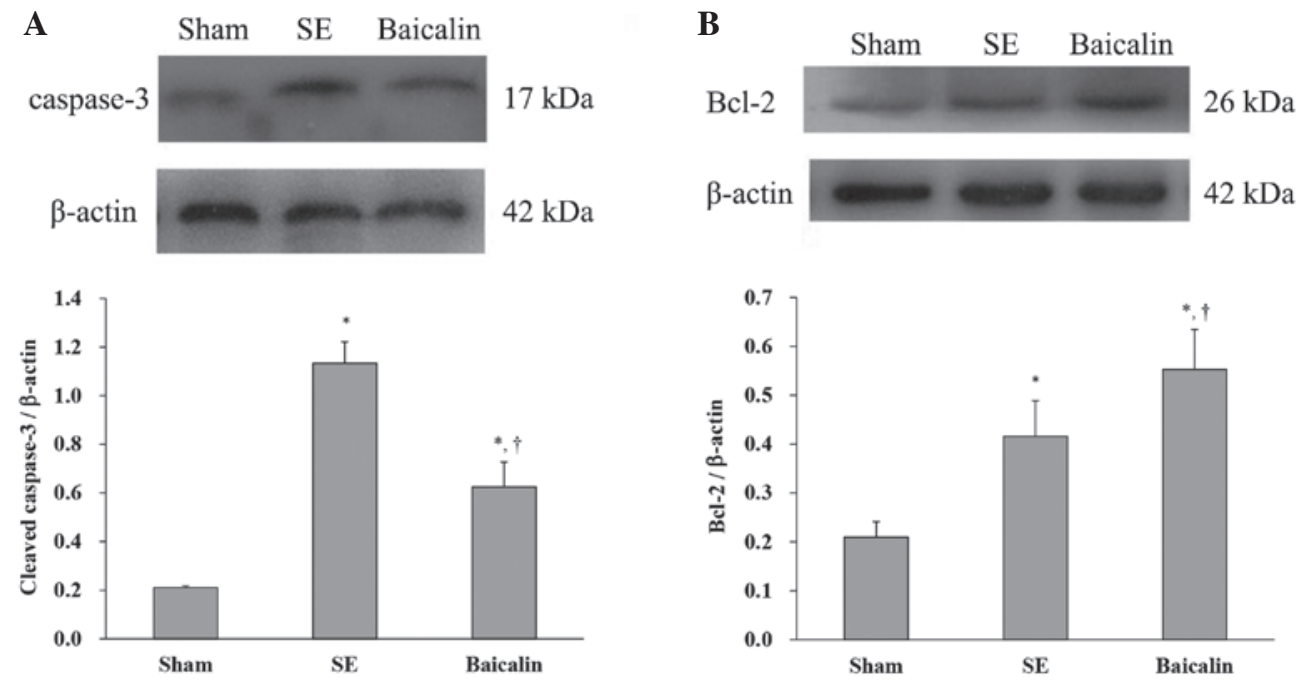

Figure 3. Protein expression of cleaved caspase-3 and Bcl-2 in the hippocampus. Effects of baicalin on the protein levels of (A) caspase-3 and (B) Bcl-2 at $72 \mathrm{~h}$ after $\mathrm{SE}$ as evidenced by western blots. Data are expressed as the ratio the respective proteins to $\beta$-actin internal control and are represented as mean \pm standard deviation ( $\mathrm{n}=6$ per group). "P<0.05 vs. sham group. ${ }^{\dagger} \mathrm{P}<0.05$ vs. SE group. Bcl-2, B-cell lymphoma; SE, status epilepticus.
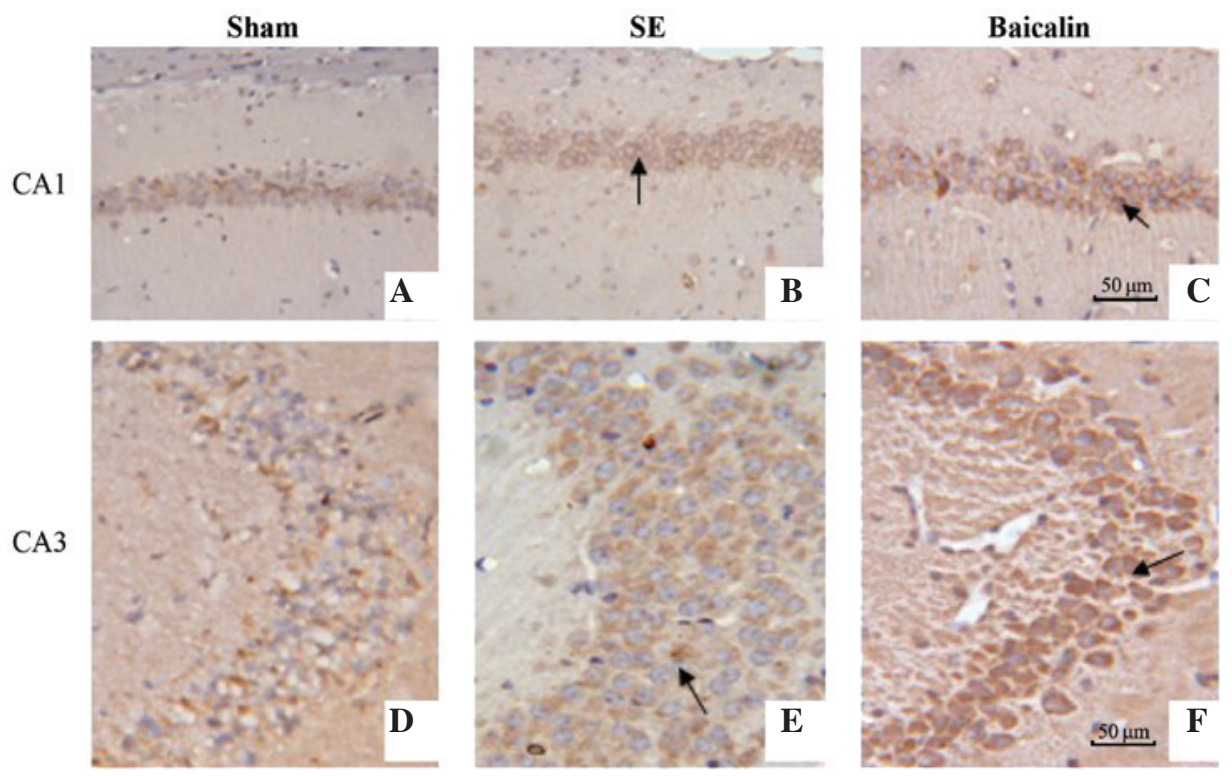

Figure 4. Photomicrographs showing the expression of B-cell lymphoma 2 (Bcl-2) positive cells in the mouse hippocampus (magnification, $\mathrm{x} 400) 72 \mathrm{~h}$ after induction of SE. (A and D) Sham control group shows low levels of Bcl-2 expression. (B and E) Numbers of Bcl-2 positive cells are increased in the post-SE mice hippocampus CA1 and CA3 regions, when compared with sham. (C and F) Activated Bcl-2 protein was higher in the hippocampal CA1 and CA3 regions in SE mice treated with baicalin when compared with the SE group. SE, status epilepticus.

Boster Biological Technology, Ltd.), transferred to nitrocellulose membranes (Membrane Solutions Co., Ltd., Shanghai, China), and blocked in 5\% nonfat dry milk buffer (Wuhan Boster Biological Technology, Ltd.). $\beta$-Actin (Cell Signaling Technology, Inc., Danvers, MA, USA) was used as a loading control. The membranes were then incubated overnight at $4^{\circ} \mathrm{C}$ with a rabbit polyclonal anti-Bcl-2 antibody (1:200; ZA-0536; Zhongshan Golden Bridge Biotechnology Co., Ltd.,) and cleaved caspase-3 (1:800; Cell Signaling Technology, Inc.), followed by incubation with horseradish-peroxidase conjugated secondary antibodies (1:2,000; SC-2004; Zhongshan Golden Bridge Biotechnology Co., Ltd.). Protein expression was detected using an enhanced chemiluminescence detection system (20-500-120; Bio-Rad Laboratories, Inc.,
Hercules, CA, USA) and exposed on X-ray film (Bio-Rad Laboratories, Inc.). The optical densities of Bcl-2 (26 kDa), cleaved caspase-3 (17 kDa) and $\beta$-actin $(42 \mathrm{kDa})$ bands on the $\mathrm{X}$-ray film were quantitatively analyzed using Quantity One software version 4.6.1 (Bio-Rad Laboratories). The results were expressed as the ratios of $\mathrm{Bcl}-2$ and cleaved caspase- 3 to $\beta$-actin.

$R N A$ extraction and reverse transcription-quantitative polymerase chain reaction ( $R T-q P C R)$. For RT-qPCR, 27 mice (9 per group) were anesthetized, then sacrificed via decapitation $12 \mathrm{~h}$ following induction of SE. The brain was carefully removed, and the hippocampus dissected. Total RNA was extracted using TRIzol reagent (Invitrogen; Thermo 

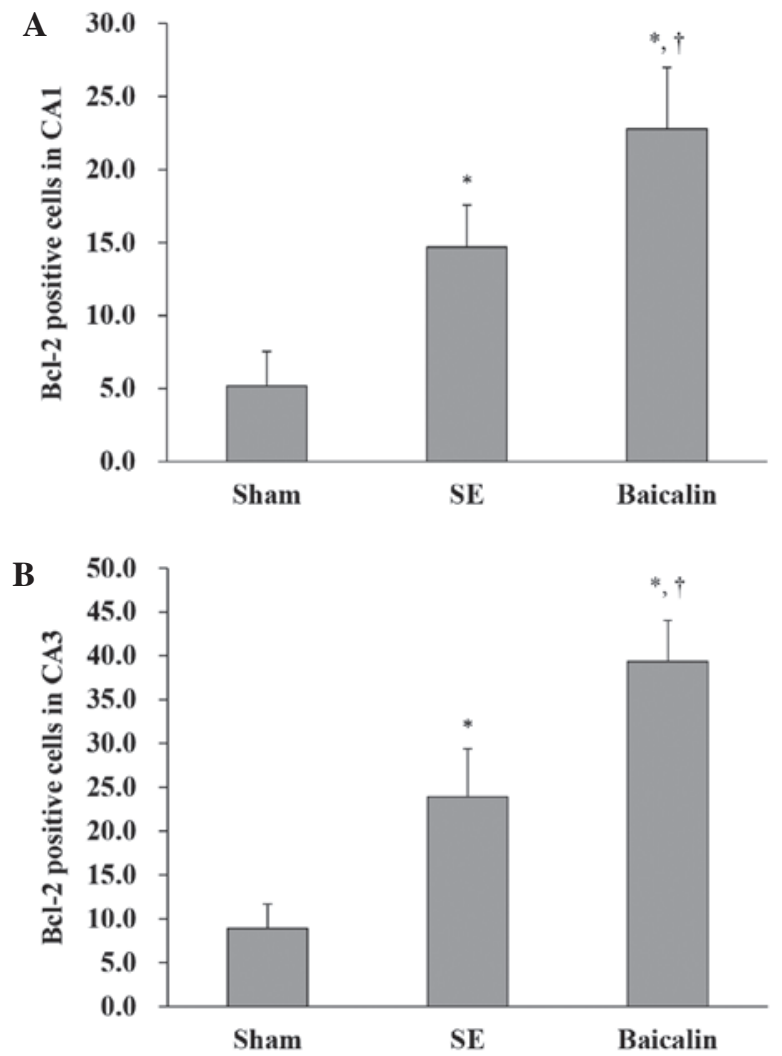

Figure 5. Quantification of Bcl-2 positive cells in hippocampus $72 \mathrm{~h}$ after induction of SE. Effects of baicalin on the number of Bcl-2 positive cells in (A) CA1 and (B) CA3 in sham, SE and baicalin treatment groups. Data are expressed as mean \pm standard deviation and compared using one-way analysis of variance with Least Significant Difference post-hoc pair-wise comparisons ( $\mathrm{n}=6$ per group). ${ }^{*} \mathrm{P}<0.05$ vs. sham group. ${ }^{\dagger} \mathrm{P}<0.05$ vs. $\mathrm{SE}$ groups. Bcl-2, B-cell lymphoma 2; SE, status epilepticus.

Fisher Scientific, Inc., Carlsbad, CA, USA), according to the manufacturer's protocol. No additional DNase treatment was conducted after the RNA was extracted from the sample. Next, $2 \mu \mathrm{g}$ total RNA underwent reverse transcription using an miR-497-specific RT-primer along with a Bulge-Loop ${ }^{\text {TM }}$ miRNA qRT-PCR Primer Set (RiboBio Co., Ltd., Guangzhou, China), according to the manufacturer's instructions. Hot start Taq polymerase as well as the forward and reverse primers for all of the genes were included in the Bulge-Loop ${ }^{\mathrm{TM}}$ miRNAqRT-PCR starter kit (RiboBio Co., Ltd.). The reaction mixture included $3.6 \mu$ l RNase-free $\mathrm{H}_{2} \mathrm{O}, 0.2 \mu \mathrm{l}$ forward and reverse primers, $5 \mu \mathrm{l}$ SYBR Green supermix and $1 \mu \mathrm{l}$ cDNA. SYBR Green I stain is maximally excited at $497 \mathrm{~nm}$, but also has secondary excitation peaks at $290 \mathrm{~nm}$ and $380 \mathrm{~nm}$. The fluorescence emission of SYBR Green I stain bound to DNA is centered at $520 \mathrm{~nm}$. PCR was conducted using a CFX-96 PCR machine (Bio-Rad Laboratories, Inc., Hercules, CA, USA), and the following conditions were used: Pre-denaturation at $95^{\circ} \mathrm{C}$ for $3 \mathrm{~min}, 40$ cycles of $95^{\circ} \mathrm{C}$ for $10 \mathrm{sec}, 60^{\circ} \mathrm{C}$ for $20 \mathrm{sec}$ and $70^{\circ} \mathrm{C}$ for $10 \mathrm{sec}$.

The ubiquitously expressed U6 small nuclear RNA was used for normalization. All primers used for qPCR were included in the commercial kit. Each sample was run in triplicate and qPCR data were analyzed using the $2^{-\Delta \Delta C q}$ method (28) and the software used to analyze the data was called BioRAD
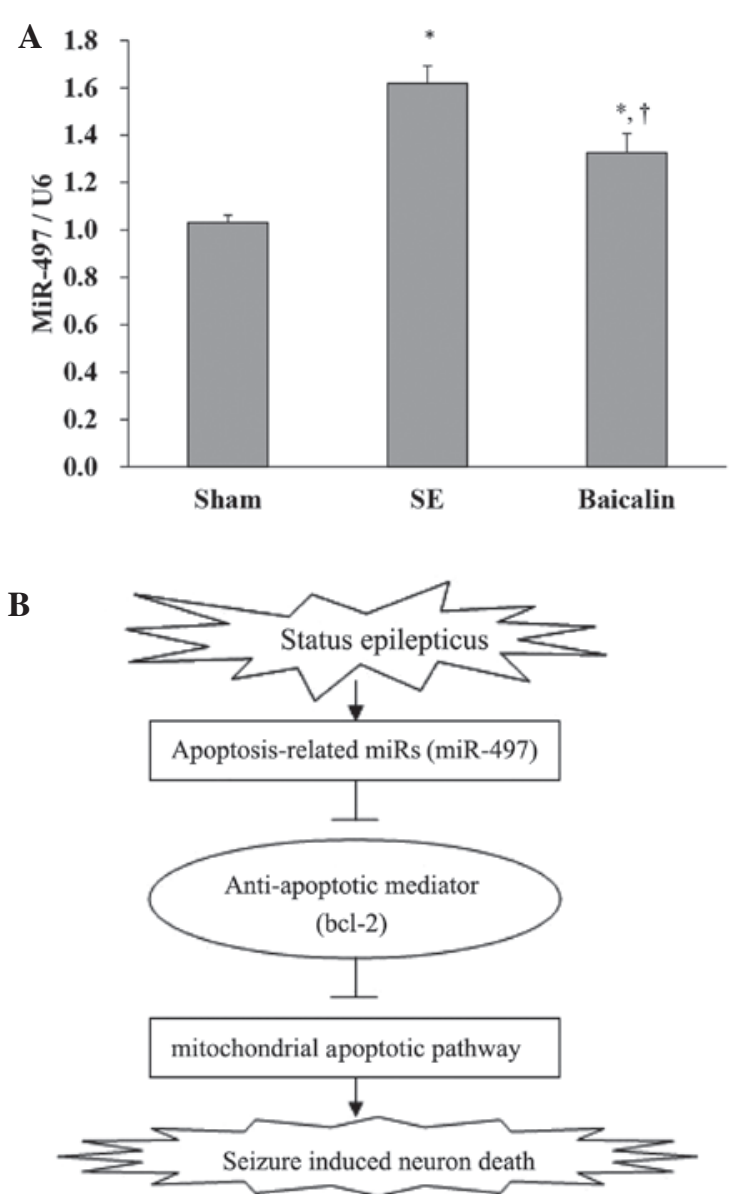

Figure 6. Quantification of mRNA expression of miR-497 in the hippocampus $12 \mathrm{~h}$ after induction of SE. (A) miR-497 expression in sham control, $\mathrm{SE}$ and baicalin groups. Expression data are represented as mean \pm standard deviation ( $\mathrm{n}=9$ per group). (B) Flow chart of proposed hypothesis. $\mathrm{P}<0.05$ vs. sham group. ${ }^{\dagger} \mathrm{P}<0.05$ vs. SE group. SE, status epilepticus; Bcl-2, B-cell lymphoma 2.

CFX Manager 2.1 (Bio-Rad Laboratories, Inc., Hercules, CA, USA).

Statistical analysis. Statistical analyses were performed using SPSS software, version 18.0 (SPSS, Inc., Chicago, IL, USA). Quantitative data were represented as mean \pm standard deviation. Differences among groups were compared using one-way analysis of variance with a Least Significant Difference post-hoc pair-wise comparison. For the apoptosis data, as the sham group had a value of zero for each sample, a one-sample t-test was applied for SE and baicalin groups to test the mean $=0$, which indicates a comparison with the sham group. Furthermore, a two-sample t-test was used to compare the difference between SE and baicalin groups. All the statistical assessments were two tailed and $\mathrm{P}<0.05$ was considered as signifying a significant difference.

\section{Results}

$S E$. The first signs of seizures occurred within $1 \mathrm{~h}$ after KA administration and continued for an additional 1-3 h. During seizures, mice displayed manifestations common to limbic SE as previously characterized by Racine et al (27) as stage I-V, including stereotyped masticatory movements (stage I), head 
movements (stage II), forelimb clonus (stage III), rearing (stage IV) and rearing and falling (stage V).

Morphological examination. Morphological examination was performed on sections cut from brains removed $72 \mathrm{~h}$ following SE onset, fixed with $4 \%$ paraformaldehyde and stained using HE. As shown in Fig. 1, the majority of neurons in the hippocampal CA1 and CA3 subfields of mice in the SE group appeared shrunken with eosinophilic cytoplasm, triangulated pyknotic nuclei and cavitation (empty holes indicative of vapor cavities; Fig. 1B and E). In the sham control group, there was no evidence of neuronal damage, with a lack of eosinophilic cytoplasm or triangulated pyknotic nuclei evident in the brains of mice in the SE group (Fig. 1A and D). Additionally, while mice that received baicalin $(100 \mathrm{mg} / \mathrm{kg})$ prior to $\mathrm{SE}$ showed eosinophilic cytoplasm and triangulated pyknotic nuclei indicative of SE induced neuronal damage, the number of damaged cells in the same areas was reduced compared to SE mice who did not receive baicalin (Fig. 1C and F).

Baicalin inhibits neuronal apoptosis after SE. TUNEL staining was used to evaluate apoptotic neuronal death in the hippocampal CA3 subfields of mice sacrificed $72 \mathrm{~h}$ following the induction of SE, processed as described, digested using proteinase $\mathrm{K}$ with chromosomal degradation visualized and using TdT followed by chromogen. Fig. 2 shows representative images of neuronal apoptosis in hippocampal CA3 subfields for each treatment group. Quantitative analysis of apoptotic nuclei as expressed as the percentage of apoptotic cells of the total revealed that the SE and baicalin groups had higher levels of TUNEL staining indicative of neuronal apoptosis compared with the sham group. By contrast, those that received baicalin treatment prior to SE had significantly lower levels of TUNEL staining than those that did not, indicating a suppression of SE-induced neuronal apoptosis (Fig. 2D) $(\mathrm{P}<0.05)$.

The levels of cleaved caspase- 3 and Bcl-2 (relative to $\beta$-actin) in brain tissue removed from mice sacrificed $72 \mathrm{~h}$ following the induction of SE was analyzed using western blotting. The results showed that SE increased cleaved caspase-3 protein expression, which was suppressed by baicalin (Fig. 3A) $(\mathrm{P}<0.05)$. By contrast, SE upregulated antiapoptotic Bcl-2 protein expression (SE, $0.42 \pm 0.07$ vs. sham, 0.21 \pm 0.03 ; $\mathrm{P}<0.001$ ) (Fig. 3B). Baicalin treatment resulted in greater increases in $\mathrm{Bcl}-2$ expression as a result of $\mathrm{SE}$ compared with $\mathrm{SE}$ alone (baicalin, $0.55 \pm 0.08$ vs. SE, $0.42 \pm 0.07 ; \mathrm{P}=0.003$ ) (Fig. 3B). These results suggest that baicalin administration following KA-induced SE leads to lower expression of proteins associated with neuronal apoptosis in the hippocampus.

Expression of Bcl-2 within the hippocampal CA1 and CA3 subfields of brain sections from mice sacrificed $72 \mathrm{~h}$ after the induction of SE was analyzed by immunohistochemistry using antibodies raised against $\mathrm{Bcl}-2$ and visualized using horseradish peroxidase/biotin labeling. Representative hippocampal images of Bcl-2 staining from the three treatment groups are shown in Fig. 4. Quantification of Bcl-2 positive cells in the CA1 (Fig. 5A) and CA3 (Fig. 5B) subfields showed that $\mathrm{SE}$ increased the number of Bcl-2 positive cells, which were further enhanced by baicalin treatment (CA1: baicalin, $22.79 \pm 4.19$ vs. $\mathrm{SE}, 14.67 \pm 2.89 ; \mathrm{P}=0.001$ ) (CA3: baicalin, $39.34 \pm 4.67$ vs. SE, $23.90 \pm 5.47 ; \mathrm{P}<0.001)$.
Baicalin inhibits the expression of miR-497. Expression of mature miR-497 was analyzed using RT-qPCR, within the CA3 and CA1 subfields of the hippocampus from mice sacrificed at $12 \mathrm{~h}$ after SE via decapitation and compared with samples from time-matched, vehicle-injected, non-seizure controls. The results showed that in the hippocampus of SE mice, expression of miR-497 was increased compared with the sham (SE, $1.62 \pm 0.32$ vs. sham, $1.03 \pm 0.15 ; \mathrm{P}<0.001$ ). However, baicalin treatment following SE reduced miR-497 expression as compared to $\mathrm{SE}$ alone (baicalin, $1.33 \pm 0.25$ vs. SE, $1.62 \pm 0.32$, $\mathrm{P}=0.02$; Fig. $6 \mathrm{~A}$ ). Thus, as shown in Fig. $6 \mathrm{~B}, \mathrm{SE}$ may induce elevation of miR-497 levels, which may negatively regulate expression of $\mathrm{Bcl}-2$.

\section{Discussion}

In the present study, intracerebral ventricle microinjection of KA was used to trigger SE, leading to the emergence of epileptic seizures and subsequent hippocampal damage. Previous studies have confirmed that apoptotic pathways contribute to seizure-induced neuronal death in this model $(4,5,24,29)$, as evidenced by significantly altered hippocampal damage in animals lacking apoptosis-associated genes and proteins, including miR-497, Bcl-2 and caspase-3. To the best of our knowledge, the present study is the first to show that baicalin not only significantly reduces the number of TUNEL-positive cells following SE, but also alters the expression of $\mathrm{Bcl}-2$ and cleaved caspase-3 protein, in addition to miR-497. However, while it may be hypothesized that baicalin exerts its neuroprotective effects via the downregulation of miR-497, leading to the upregulation of the caspase-3 inhibitor Bcl-2, the precise mechanisms underlying the action of baicalin remain poorly understood and will require further study.

The present finding that SE increases Bcl-2 expression in the hippocampal CA1 and CA3 regions is consistent with a previous report (30). It was also found that baicalin significantly enhanced the number of cells expressing Bcl-2, while reducing miR-497 expression and caspase-3 protein activation. To our knowledge, this is the first demonstration of the efficacy of baicalin in enhancing the effect of SE on Bcl-2 expression while reducing caspase- 3 cleavage.

In this study, we reported that miR-497 is rapidly upregulated in the hippocampus following KA induced SE in mice, demonstrating that miR-497 may be associated with early pathological changes and seizure-induced neuronal death. As miR-497 is known to downregulate the expression of Bcl-2 (14,15), SE-induced expression of miR-497 may promote apoptosis by limiting post SE increases in Bcl-2 levels, as Bcl-2 has a well-established inhibitory effect on caspase-3 activation (8). However, it was presently found that baicalin inhibited SE-induced increases in miR-497 expression and enhanced SE-induced increases in Bcl-2 protein levels. Therefore, we hypothesize that the suppression of caspase-3 activation by baicalin is mediated by the inhibition of the expression of miR-497, subsequently disinhibiting the expression of the caspase-3 inhibitor Bcl-2.

In conclusion, the present study demonstrated that baicalin exerts a neuroprotective effect against apoptotic damage as a result of KA-induced epileptic seizures. This study presents 
further support to the potential use of baicalin as a therapeutic agent to reduce seizure-induced brain injury. We hypothesize that baicalin may limit delayed neuronal death by preventing miR-497 from inhibiting Bcl-2 expression, which leads to decreased levels of cleaved caspase-3 protein and subsequent apoptosis in epileptic insults. While it has previously been shown that miR-497 regulates neuronal death following ischemia (23), the present study is the first to identify an association between miR497 and seizure-induced damage. However, additional studies will be necessary to determine the precise mechanisms involved in the impact of baicalin on seizure-induced neuronal death. Furthermore, additional studies are required to determine effect of baicalin on normal controls, thereby determining if its effect is non-specific or directly related to SE. This study further demonstrates the role of miRs in regulating the antiapoptotic Bcl-2 family proteins and proapoptotic caspase family proteins.

\section{Acknowledgements}

This study was supported by the Natural Science Foundation of Fujian Province, China (grant no. 2011J01175), and by internal funding from the Department of Neurosurgery, Union Hospital of Fujian Medical University.

\section{References}

1. Henshall DC and Simon RP: Epilepsy and apoptosis pathways. J Cereb Blood Flow Metab 25: 1557-1572, 2005.

2. Lopez-Meraz ML, Niquet J and Wasterlain CG: Distinct caspase pathways mediate necrosis and apoptosis in subpopulations of hippocampal neurons after status epilepticus. Epilepsia 51 (Suppl 3): S56-S60, 2010.

3. Elmore S: Apoptosis: A review of programmed cell death. Toxicol Pathol 35: 495-516, 2007.

4. Kondratyev A and Gale K: Intracerebral injection of caspase-3 inhibitor prevents neuronal apoptosis after kainic acid-evoked status epilepticus. Brain Res Mol Brain Res 75: 216-224, 2000.

5. Henshall DC, Araki T, Schindler CK, Lan JQ, Tiekoter KL, Taki W and Simon RP: Activation of Bcl-2-associated death protein and counter-response of Akt within cell populations during seizure-induced neuronal death. J Neurosci 22: 8458-8465, 2002.

6. Uysal H, Cevik IU, Soylemezoglu F, Elibol B, Ozdemir YG, Evrenkaya T, Saygi S and Dalkara T: Is the cell death in mesial temporal sclerosis apoptotic? Epilepsia 44: 778-784, 2003.

7. Hua F, Cornejo MG, Cardone MH, Stokes CL and Lauffenburger DA: Effects of Bcl-2 levels on Fas signaling-induced caspase-3 activation: Molecular genetic tests of computational model predictions. J Immunol 175: 985-995, 2005.

8. Swanton E, Savory P, Cosulich S, Clarke P and Woodman P: $\mathrm{Bcl}-2$ regulates a caspase-3/caspase-2 apoptotic cascade in cytosolic extracts. Oncogene 18: 1781-1787, 1999.

9. Ambros V: microRNAs: Tiny regulators with great potential. Cell 107: 823-826, 2001

10. Bartel DP: MicroRNAs: Genomics, biogenesis, mechanism, and function. Cell 116: 281-297, 2004.

11. Barak B, Shvarts-Serebro I, Modai S, Gilam A, Okun E, Michaelson DM, Mattson MP, Shomron N and Ashery U: Opposing actions of environmental enrichment and Alzheimer's disease on the expression of hippocampal microRNAs in mouse models. Transl Psychiatry 3: e304, 2013.
12. Jeyaseelan K, Lim KY and Armugam A: MicroRNA expression in the blood and brain of rats subjected to transient focal ischemia by middle cerebral artery occlusion. Stroke 39: 959-966, 2008.

13. Yadav S, Pandey A, Shukla A, Talwelkar SS, Kumar A, Pant AB and Parmar D: miR-497 and miR-302b regulate ethanol-induced neuronal cell death through BCL2 protein and cyclin D2. J Biol Chem 286: 37347-37357, 2011.

14. Yin KJ, Deng Z, Huang H, Hamblin M, Xie C, Zhang J and Chen YE: miR-497 regulates neuronal death in mouse brain after transient focal cerebral ischemia. Neurobiol Dis 38: 17-26, 2010.

15. Zhu W, Zhu D, Lu S, Wang T, Wang J, Jiang B, Shu Y and Liu P: miR-497 modulates multidrug resistance of human cancer cell lines by targeting BCL2. Med Oncol 29: 384-391, 2012.

16. Hu K, Zhang C, Long L, Long X, Feng L, Li Y and Xiao B: Expression profile of microRNAs in rat hippocampus following lithium-pilocarpine-induced status epilepticus. Neurosci Lett 488: 252-257, 2011

17. Kan AA, van Erp S, Derijck AA, de Wit M, Hessel EV, O'Duibhir E, de Jager W, Van Rijen PC, Gosselaar PH, de Graan PN and Pasterkamp RJ: Genome-wide microRNA profiling of human temporal lobe epilepsy identifies modulators of the immune response. Cell Mol Life Sci 69: 3127-3145, 2012.

18. Pitkänen A and Lukasiuk K: Molecular biomarkers of epileptogenesis. Biomark Med 5: 629-633, 2011.

19. Cheng F, Lu Y, Zhong X, Song W, Wang X, Sun X, Qin J, Guo S and Wang Q: Baicalin's therapeutic time window of neuroprotection during transient focal cerebral ischemia and its antioxidative effects in vitro and in vivo. Evid Based Complement Alternat Med 2013: 120261, 2013.

20. Li L, Bao H, Wu J, Duan X, Liu B, Sun J, Gong W, Lv Y, Zhang $\mathrm{H}$, Luo Q, et al: Baicalin is anti-inflammatory in cigarette smoke-induced inflammatory models in vivo and in vitro: A possible role for HDAC2 activity. Int Immunopharmacol 13: 15-22, 2012.

21. Cao Y, Mao X, Sun C, Zheng P, Gao J, Wang X, Min D, Sun H, Xie N and Cai J: Baicalin attenuates global cerebral ischemia/reperfusion injury in gerbils via anti-oxidative and anti-apoptotic pathways. Brain Res Bull 85: 396-402, 2011.

22. Chen J, Li Z, Chen AY, Ye X, Luo H, Rankin GO and Chen YC Inhibitory effect of baicalin and baicalein on ovarian cancer cells. Int J Mol Sci 14: 6012-6025, 2013.

23. Tu XK, Yang WZ, Shi SS, Wang $\mathrm{CH}$ and Chen CM: Neuroprotective effect of baicalin in a rat model of permanent focal cerebral ischemia. Neurochem Res 34: 1626-1634, 2009.

24. Ouyang LQ, Liang RS, Yang WZ, Chen CM, Tu XK and Wen S: Neuroprotective effect of Baicalin on hippocampal neurons after status epilepticus in mice. Zhong Hua Shi Yan Wai Ke Za Zhi She 29: 1697-1699, 2012 (In Chinese).

25. Gröticke I, Hoffmann K and Löscher W: Behavioral alterations in a mouse model of temporal lobe epilepsy induced by intrahippocampal injection of kainate. Exp Neurol 213: 71-83, 2008.

26. Laursen SE and Belknap JK: Intracerebroventricular injections in mice. Some methodological refinements. J Pharmacol Methods 16: 355-357, 1986.

27. Racine RJ, Steingart M and McIntyre DC: Development of kindling-prone and kindling-resistant rats: Selective breeding and electrophysiological studies. Epilepsy Res 35: 183-195, 1999.

28. Livak KJ and Schmittgen TD: Analysis of relative gene expression data using real-time quantitative PCR and the 2(-Delta Delta C(T)) Method. Methods 25: 402-408, 2001.

29. Pretel S, Applegate CD and Piekut D: Apoptotic and necrotic cell death following kindling induced seizures. Acta Histochem 99: 71-79, 1997.

30. Cheng JY, Wu LN, Wang QZ, Gan YF, Liu GY and Yu H: Altered mitochondria and Bcl-2 expression in the hippocampal CA3 region in a rat model of acute epilepsy. Zhong Guo Shen Jing Zai Sheng Yan Jiu Ying Wen Ban 4: 276-280, 2009 (In Chinese). 